\title{
SONOCISTOGRAFIA: ESTUDIO COMPARATIVO CON URETROCISTOGRAFIA CONVENCIONAL
}

\author{
Drs. Marcela Cortés S, José D. Arce V, Víctor Díaz B.
}

Servicio de Radiología. Clínica Santa María.

\begin{abstract}
Vesicoureteral reflux was studied in seventeen (17) children with echo-enhanced cystosonography. Results were compared with voiding cystography with digital technique as a gold standard. The sensitivity and predictive positive value were $94.3 \%$. The specificity and predictive negative value were $93.7 \%$. The sonocistography presents as a good alternative to voiding cystography. This technique is especially useful in children on account of their pathology and the fact that they are exposed to multiple ionizing radiation examinations.
\end{abstract}

Key words: Bladder, Ultrasound, Contrast media, Ureter reflux.

Resumen: Se efectuó estudio de reflujo vesicoureteral en 17 niños empleando ultrasonografía con ecorrealzador (sonocistografía) y se compararon sus resultados con cistografía radiológica con técnica digital como estándar dorado. La sensibilidad y el valor predictivo positivo medidos de esta forma fueron de $94.4 \%$ y los valores predictivos negativos y especificidad de $93.7 \%$. El uso de sonocistografía es una alternativa valida en el control de reflujo vesicoureteral y al no emplear radiación ionizante estaría especialmente indicada en niños que por su patología de base son sometidos a múltiples exámenes radiológicos.

Palabras claves: Reflujo vesicoureteral, Cistografía, Ecorrealzador, Ultrasonografía.

\section{Introducción}

Los métodos de imagen usados para investigar reflujo vesicoureteral (RVU) son la cistografía radiológica (C-Rx) o la cistografía con radioisótopos, ambas técnicas usan radiación ionizante ${ }^{(1,2)}$.

El estudio radiológico con técnica digital y optimización para reducción de la dosis, en niños en

Cortés M, Arce JD, Díaz V. Sonocistografía: Estudio comparativo con uretrocistografía convencional. Rev Chil Radiol 2002; 8: 24-27.

Correspondencia: Dra. Marcela Cortés S. Clínica Santa María. Avenida Santa María 0410. Providencia. Santiago de Chile. el rango de edad de 1 a 5 años, expone a los ovarios a una dosis de 0,002-0,003 Gy y a los testículos a una dosis de 0,001-0,003 $\mathrm{Gy}^{(1)}$. La técnica cintigráfica, aun cuando baja en forma importante la dosis de radiación gonadal, no da buen detalle anatómico.

El ultrasonido es una técnica de imagen que no usa radiación ionizante y es bien aceptada por el paciente pediátrico.

El objetivo de este trabajo es evaluar y proponer como alternativa diagnóstica, en el estudio de RVU en niños, el uso de ultrasonografía con ecorrealzador.

\section{Material y método}

El estudio se efectuó en 17 niños (13 femenino y 4 masculino), con un total de 34 unidades ureterorrenales. Las edades variaron desde 1 hasta 12 años (promedio: 5 años 6 meses).

Las indicaciones médicas para realizar el examen radiológico fueron: infección del tracto genitourinario en 6 pacientes $(35,2 \%)$, seguimiento de RVU previamente diagnosticado en 7 pacientes $(41,2 \%)$, malformaciones congénitas y síndromes asociados en 4 pacientes (23,5\%).

A los padres o tutores, que acompañaban a los niños, se les explicó el procedimiento radiológico y ultrasonográfico, obteniendo el consentimiento de cada uno de ellos.

Los 8 primeros pacientes fueron examinados por el mismo operador, quien realizó el estudio radiológico (C-Rx) y ultrasonográfico (sonocistografía: C-US). En los otros 9 niños; un radiólogo les realizó primero el estudio radiológico y luego otro examinador la C-US, sin conocimiento previo de los resultados obtenidos en la C-Rx.

Los exámenes radiológicos fueron ejecutados en equipos Siemens Digital Sireskop. Los estudios ultrasonográficos en equipos ATL 3000 y 5000 con transductores convexos de banda ancha de $7-4 \mathrm{MHz}$.

La vejiga se cateterizó transuretralmente bajo procedimiento aséptico, realizándose el estudio radiológico convencional, luego sin retirar la sonda se efectuó el estudio ultrasonográfico.

Previo a la inyección del ecorrealzador, se rea- 
lizó una evaluación morfológica de ambas unidades ureterorenales, riñones y vejiga.

Para la C-US se infundió suero fisiológico en un volumen estimado de acuerdo a la capacidad vesical obtenida en la cistografía. Posteriormente se instiló $1 \mathrm{mg} / \mathrm{kg}$ de ecorrealzador diluido en $10 \mathrm{ml}$ de agua estéril (Levovist $2,5 \mathrm{~g}, 300-200 \mathrm{mg} / \mathrm{ml}$ en solución compuesta por $99,9 \%$ de galactosa y $0,1 \%$ de ácido palmítico. Levovist SHU 508 A, Schering, Berlín, Alemania). Resulta de esta forma una solución con una osmolalidad que a $37^{\circ} \mathrm{C}$ es de $1175 \mathrm{mmol} / \mathrm{kg}$, ello representa lo mínimo capaz de ser detectado como señal en ultrasonido en el tracto genitourinario después de 90 segundos de equilibrio logrado por batido homogéneo de la suspensión ${ }^{(3)}$. El ecorrealzador diluido en suero fisiológico produce un contraste homogéneo e hiperecogénico en la vejiga (Figura 1).

La dilución excesiva de Levovist reduce la señal detectable sobre todo en presencia de dilatación del tracto urinario(4).

Se evaluó con ultrasonido en escala de grises durante al menos dos ciclos miccionales. Primero la vejiga y uniones vesicoureterales a nivel de los
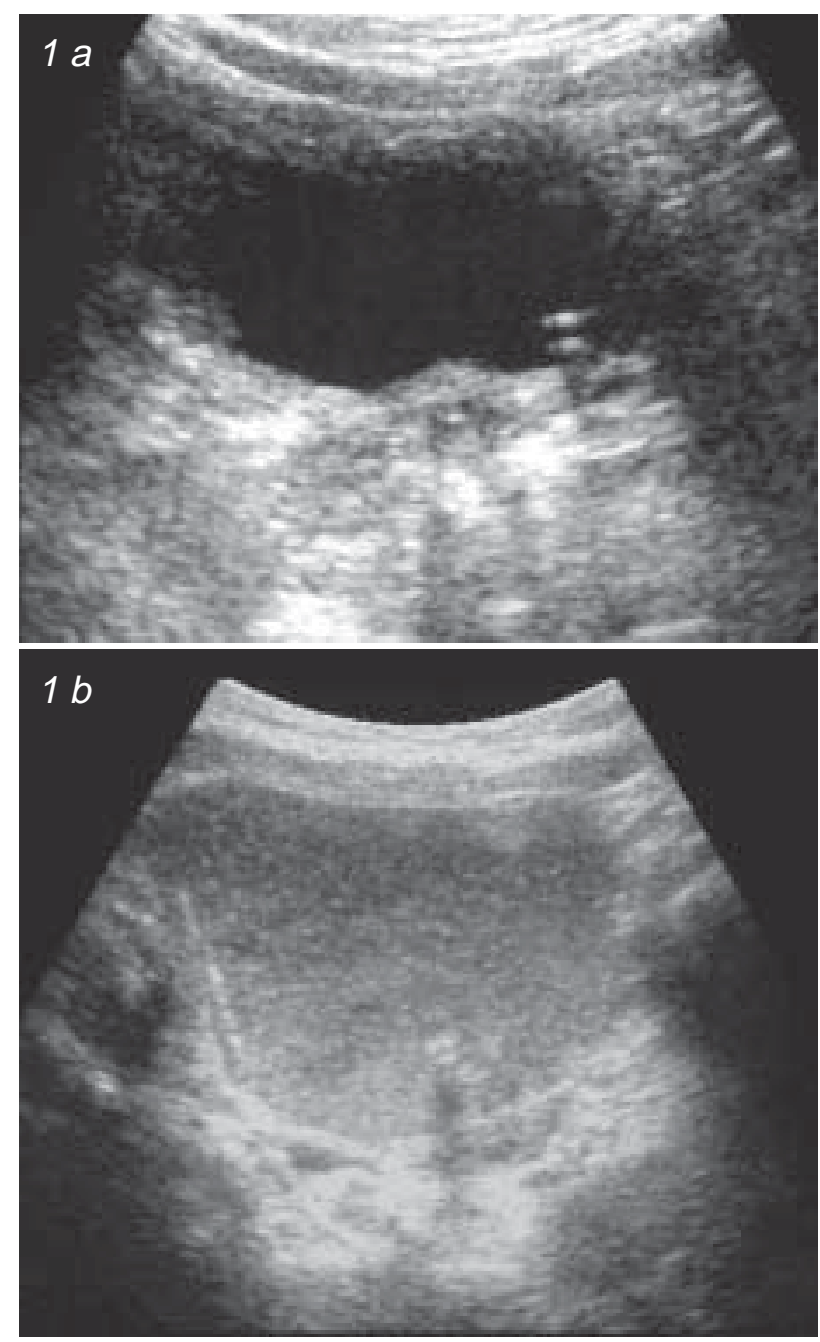

Figura 1a,b. Ecorrealzador en vejiga. a. Vejiga con suero b. Vejiga con ecorrealzador hiperecogenico. meatos y luego en forma comparativa ambos riñones en posición decúbito supino, con cortes longitudinales y transversales, registrando las imágenes, en un comienzo como imágenes basales y luego los cambios posteriores al llene vesical con el ecorrealzador.

EI RVU documentado en el estudio con ultrasonido fue considerado positivo cuando se observó la presencia de microburbujas del ecorrealzador en uréteres retrovesicales o en el tracto genitourinario superior, incluido uréter proximal y pelvis renal (cálices y pelvis).

Los parámetros comunes usados como prueba diagnósticos (sensibilidad, especificidad, valor predictivo positivo y valor predictivo negativo) fueron calculados usando la uretrocistografía miccional como estándar dorado de referencia (Galen-Gambine análisis) ${ }^{(5)}$.

\section{Resultados}

Cuando hubo reflujo de ecorrealzador al tracto urinario superior (sistemas pielocalicilares) éste fue fácilmente detectado (Figura 2) y diferenciado del gas intestinal, esta observación fue más difícil en la porción distal de los uréteres. En algunos de nuestros casos se visualizó distensión de la pelvis renal sin observar en ella la presencia de microburbujas, ello no fue considerado como reflujo solo un fenómeno secundario a ectasia.

Todos los pacientes toleraron bien el examen y no se registraron complicaciones durante el examen o en el período posterior a él.

La C-Rx fue usada como estándar dorado de referencia en la evaluación de sensibilidad, especificidad y en los valores predictivos de la cistosonografía. Un paciente tuvo reflujo bilateral.

Usando los resultados de normal (sin RVU) y anormalidad (con RVU) se compararon ambos métodos (tabla I). Así la sensibilidad y valor predictivo positivo obtenidos fueron de $94,4 \%$ cada una y los valores predictivos negativo y especificidad fueron cada uno de $93,7 \%$.

Los resultados en cuanto al grado de RVU en la C-Rx se clasificaron de acuerdo al sistema internacional( $^{(6)}$ (tabla II). En la C-US no se evaluó grado de

Tabla I : Cistografía Rx versus Sonocistografía

\begin{tabular}{|c|c|c|c|}
\hline \multicolumn{4}{|c|}{$C-R x$} \\
\hline C-US & ANORMAL & NORMAL & TOTAL \\
\hline ANORMAL & 17 & 1 & 18 \\
\hline NORMAL & 1 & 15 & 16 \\
\hline TOTAL & 18 & 16 & 34 \\
\hline
\end{tabular}



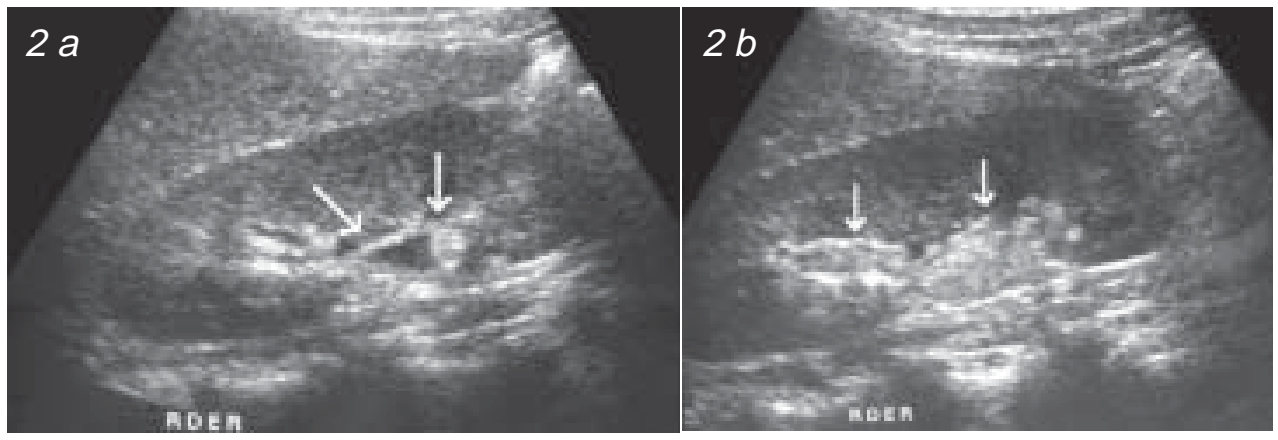

Figura 2 a,b,c. Reflujo vesicoureteral derecho. a. Riñón derecho previo al uso de ecorrrealzador. Pelvis renal con pequeña cantidad de orina (flechas). b. Riñón derecho después del uso de ecorrealzador. Pelvis renal con ecorrealzador (flechas). c. Cistografía

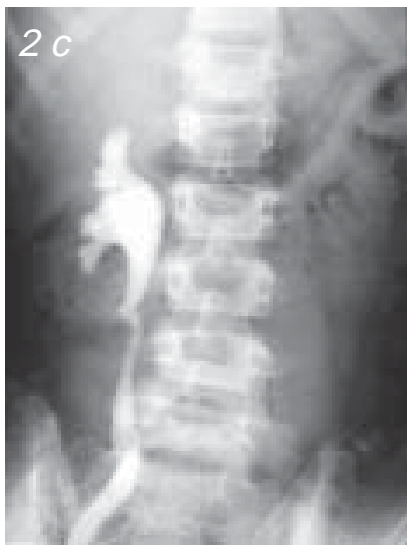
Radiológica.

RVU, sólo se registró su presencia o ausencia como anormal o normal. Se caracterizó además la morfología de los riñones, uréteres y vejiga, observándose distensión de la pelvis renal (dos casos) o ectasia piélica (cuatro casos) sin detectarse reflujo del ecorrealzador durante el ciclo miccional, ni del medio de contraste en la C-Rx.

En un caso se identificó reflujo (grado II) en la C-Rx y sólo ectasia piélica en la C-US. En otro caso se registró lo inverso existiendo reflujo del ecorrealzador, al sector proximal del uréter; sin observarlo en el estudio radiológico, en ambos casos se trató de reflujos de bajo grado.

\section{Discusión}

EI RVU es un proceso dinámico y un importante porcentaje de ellos, especialmente los reflujos de bajo grado, se resuelven espontáneamente durante el desarrollo ${ }^{(7)}$. Los exámenes de seguimiento son necesarios para la evaluación del tratamiento.

La detección de RVU de alto grado por ultrasonido ha sido descrita desde $1981^{(8)}$. Salih et al. ${ }^{(9)}$ demostró la visualización del fenómeno de "jet" en reflujo de bajo grado con Doppler color.

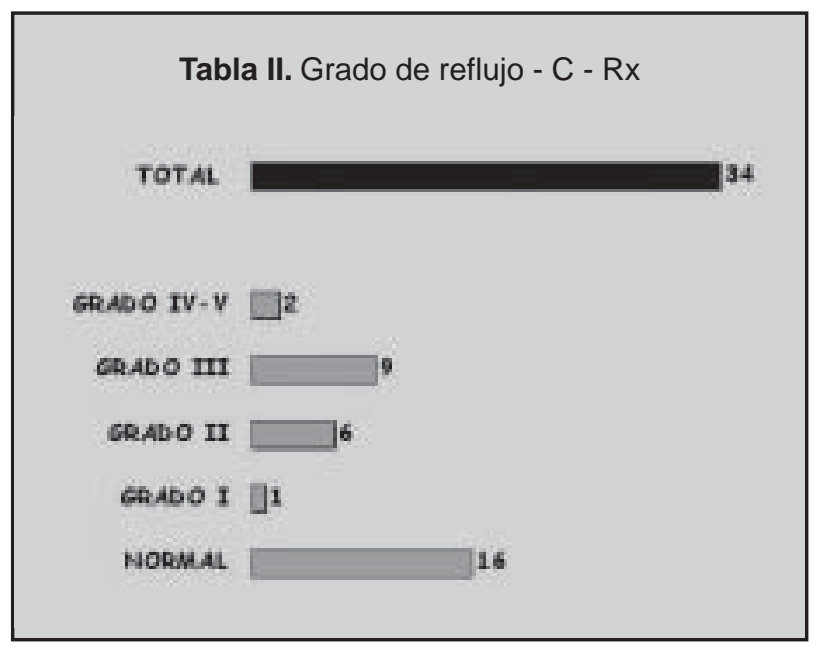

La detección de reflujo con ultrasonido ha sido también reportada usando microburbujas de aire en solución salina o agentes de contraste yodados ${ }^{(10,11)}$. Sin embargo, esos métodos tienen un tiempo limitado de observación para evaluar reflujo, debido a la rápida disolución de las microburbujas formadas en la solución utilizada. Los agentes de contraste o ecorrealzadores son más útiles en la detección de RVU, no sólo por su hiperecogenicidad, sino por el tiempo que permanecen estables en el tracto urinario, permitiendo un adecuado tiempo de examen de la vejiga, uréteres y riñones ${ }^{(12)}$.

La albúmina de los ecorrealzadores produce una ecogenicidad uniforme, fácilmente diferenciada de la del gas intestinal. Las microesferas aparecen adheridas a la pared del uréter, vejiga y pelvis renal, permaneciendo estable hasta después de 30 minutos $^{(13)}$.

La desventaja del uso de ecorrealzadores en la detección de RVU es que no permite el estudio de la uretra, lo que es necesario para excluir un reflujo secundario a alteración de la unión ureterovesical o por la presencia de valvas uretrales. Ello es importante de descartar en todos los pacientes con reflujo de alto grado o en neonatos de sexo masculino con infección de tracto urinario con o sin hidronefrosis. Otro inconveniente asociado es el alto costo relativo del ecorrealzador comparado con los agentes de contraste radiológicos.

El caso donde se observó RVU, sólo en C-US y detectado en la C-Rx, se pueden explicar por el hecho que el reflujo es un fenómeno intermitente, hallazgo conocido, motivo por el cual se realiza comúnmente al menos dos ciclos miccionales en el examen de rutina particularmente en lactantes. Otros autores describen falsos positivos en el estudio ultrasonográfico de reflujo especialmente aquellos de bajo grado(4,9,14). La posibilidad de observar los riñones, uréteres y la vejiga por un periodo continuo y mayor al del estudio radiológico podría explicar estos hallazgos ${ }^{(4,11)}$. 


\section{Conclusión}

El estudio ultrasonográfico con ecorrealzadores es una técnica útil y segura en la detección de RVU, con sensibilidad y especificidad satisfactorias de $94,4 \%$ y 93,7 respectivamente, incluyendo además la demostración de otras anomalías asociadas de tracto genitourinario. Es posible el estudio dinámico de la dilatación de cálices y de la pelvis renal en reflujos de bajo grado, además permite la evaluación del parénquima renal (grosor cortical, ecogenicidad, diferenciación córticomedular y tamaño renal).

Sus eventuales limitaciones nos hacen plantearlo más bien para el control de pacientes con reflujo conocido, en especial en aquellos pacientes que por su patología de base son sometidos a numerosos estudios radiológicos.

\section{Bibliografía}

1. Cleveland $\mathrm{RH}$, Constantinou C, Blikman JG, et al Voiding cystography in children: value of digital fluoroscopy in reducing radiation. AJR 1992; 158: 137142.

2. Lowry PA, Pjura GA, Kim EE, Brown WD. Radionuclide imaging of lower genitourinary tract. In: Gottschalk A, Hoffer PB, Potchen EJ, Berger HJ (eds). Diagnotic Nuclear Medicine, $2^{\text {nd }}$ edn. Baltimore, 1988; Williams \& Wilkins, pp 967-984.

3. Mentzel HJ, Vogt S, Patzer L, Schubert R, John U, Misselwitz J, Kaiser W. Contrast-Enhacement sonography of vesicoureteral reflux in children: Preliminary results. AJR 1999; 173: 737-740.

4. Maurizio Bosio. Cystography wiht echocontrast: a new imaging modality to detect vesicoureteric reflux in children. Pediatr Radiol 1998; 28: 250-255.

5. Galen RS, Gambine RS. Beyond normaly: the predictive value and efficiecy of medical dianosis. New York. Wiley 1975.

6. Lebowitz RL, OlblingH, Parkkulainen KU, Smellie JM, Tamminen-Mobius TE. International system of radiograghpic grading of vesicoureteric reflux. Pediatr Radiol 1985; 15: 105-109.

7. Ashcraft KW. Vesicoureteric reflux. In: Ashcraft KW, ed. Pediatric urology. Philadelphia: Saunders, 1990;151173.

8. Hofmann V. Ultraschalldiagnostik bei vesiko-ureteralem Reflux. Z Urol Nephrol 1981; 74: 39-261.

9. Salih M, Baltaci S, Kilic S, Anafarta K, Bedük Y. Color flow Doppler sonography in the diagnosis of vesicoureteric reflux. Pediatr Urol 1994; 26: 93-97.

10. Kessler R, Altman D.H. Real time sonographic detection of vesicoureteral reflux in children. AJR 1982; 138: 1033.

11. Hanbury DC, Coulden RA, Farman P, Sherwood T. Ultrasound cystography in the diagnosis of vesicouretreric reflux. Brit J Urol 1990; 65: 250.

12. Atala A, Wible JH, Shae JC, Carr MC, RetikAB, Mandell $J$. Sonography with sonicated albumin in the deteccion of vesicoureteral reflux. J Urol 1993; 150 :756-758.

13. Atala A, Ellsworth P, Share J, Paltiel H, Walker Rd, Retik A. Comparition of sonicated albumin enhanced sonography to fluoroscophy for detection vesicouretral reflux. J Urol 1998; 160:1820-1822.

14. Darge K, Duetting T, Zieger B, Moehring K, Troeger J. Diagnosis of vesicoureteral reflux with echoenhacement voiding urosonography. Radiology 1998; 38: 405-409. 